\title{
Mining Students Pre-instruction Beliefs for Improved Learning
}

\author{
Ángel Pérez-Lemonche \\ MIT \\ Cambridge, MA 02139 \\ angelpl@mit.edu
}

\author{
Rachel Henderson \\ Michigan State university \\ East Lansing, MI 48823 \\ rjhenderson127@gmail.com
}

\author{
John Stewart \\ West Virginia University \\ Morgantown, WV \\ 26506-6315 \\ jcstewart1@mail.wvu.edu \\ Alex Shvonski \\ MIT \\ Cambridge, MA 02139 \\ shvonski@mit.edu
}

\author{
Byron Drury \\ MIT \\ Cambridge, MA 02139 \\ bdrury@MIT.edu
}
David E. Pritchard MIT
Cambridge, MA 02139
dpritch@mit.edu

\begin{abstract}
In principle, learning can be increased by assessing the detailed state of student knowledge and mistaken knowledge with a pre-test and then optimizing instruction as measured by the post-test score. As a first step in this direction, we applied a Multidimensional Item Response Theory (MIRT) to 17,000 pre-instruction administrations of the Force Concept Inventory (FCI) to study students' initial knowledge in detail. Examination of Item Response Curves (IRCs) showed that even students scoring below chance are not randomly guessing, but instead preferentially select only one or two distractors. Two dimensional IRT applied to the entire set of 150 possible responses, rather than applied dichotomously to the thirty questions, revealed two skill dimensions of comparable variance. Perpendicular directions were identified within this space corresponding to Newtonian ability and propensity to select responses whose IRC's have a maximum at intermediate Newtonian ability rather than at the top of bottom of this dimension. These intermediate responses corresponded to known pre-Newtonian ideas, particularly the Medieval concept of impetus. The ability to measure the detailed misconceptions of individual students or classes will allow development and application of instructional interventions for such specific misunderstandings, which are typically unchanged by traditional instruction.
\end{abstract}

Permission to make digital or hard copies of part or all of this work for personal or classroom use is granted without fee provided that copies are not made or distributed for profit or commercial advantage and that copies bear this notice and the full citation on the first page. Copyrights for third-party components of this work must be honored. For all other uses, contact the Owner/Author.

L@S '19, June 24-25, 2019, Chicago, IL, USA

(C) 2019 Copyright is held by the owner/author(s).

ACM ISBN 978-1-4503-6804-9/19/06.

DOI: $10.1145 / 3330430.3333637$

\section{Author Keywords}

Assessing Learning, Response Curves, pre-post testing, Multidimensional IRT, Force Concept Inventory, Newtonian

\section{ACM Classification Keywords}

H.5.m. Information interfaces and presentation (e.g., HCI): Miscellaneous; See http://acm.org/about/class/1998 for the full list of ACM classifiers. This section is required.

\section{INTRODUCTION}

One big promise of learning at scale is to improve learning by studying student knowledge in detail and adjusting future instruction according to the results. Arguably the best way to measure learning is through pre-post testing, using research-developed instruments designed to measure what it is desired for students to learn. The pioneering such instrument in physics education research measures conceptual rather than calculational ability - the Force Concept Inventory (FCI) which was designed to measure student learning of the basic Newtonian concepts of force and motion (Hestenes, Wells, \& Swackhamer, 1991).

Physics instructors worldwide were stunned by two major revelations that followed immediately upon administering the FCI. The first was that students entering the introductory physics classroom in college typically answered only $\sim 1 / 3$ of the questions using Newtonian reasoning on the pretest. The second was that conventional lecture-based instruction remedied somewhat less than 1/4 of the non-Newtonian ideas that the students brought into the classroom, leaving the majority of students well short of the $70 \%$ regarded as the Newtonian threshold on the post-test. The second was most disturbing because instructors thought that they were teaching these concepts to their students.

More recently, education researchers have shown that today's students bring many alternate conceptions to bear in understanding force, motion, and other physics concepts. These center on the relationship between force and motion, but include deeper cognitive ideas such as 
phenomenological primitives (Hammer, 1996; diSessa \& Sherin, 1998) and ontological categories (Gupta, Hammer, $\&$ Redish, 2010).

By and large, the FCI has been administered pre- and postinstruction, the results often being interpreted using the score improvement normalized by the maximum possible improvement (Hake, 1998) which is a measure of the quality of the instruction that is surprisingly unaffected by the initial level of the students. Many techniques and pedagogies have been developed with the specific objective of raising the post-instruction score, both relative to the pre-score and overall. The central conclusion is that various methods of interactive instruction increase the fraction of non-Newtonian ideas that are replaced by Newtonian concepts from $\sim 1 / 4$ to $<\sim 1 / 2$ (Hake, 1998), which begs for further improvement.

We maintain that significant additional improvement must involve a measurement of the particular non-Newtonian ideas that an individual student or class has, followed by instruction tailored to address these alternate conceptions. It is well known that such alternative conceptions are firmly held beliefs Clement, J. (1982), and various methods have been developed to persuade students to abandon them, for example Mazur, E. (1999), Sokoloff, D. R., \& Thornton, R. K. (1997). This raises the challenge of finding out what specific alternative conceptions are held by those beginning instruction in order that they may be directly addressed through instruction.

Our approach to the determination of the particular alternate conceptions held by entering students is based on analysis of the particular wrong (i.e. non-Newtonian) responses selected by the students. Since these distractors in the FCI were designed to represent both common sense concepts and historical concepts about force and motion, it should be possible to determine the particular set of distractors selected by an individual or a class and from them to identify a smaller set of specific alternative conceptions, or a more general student belief system, that can then be remediated through targeted instruction.

The main emphasis in previous studies of the FCI has been in classifying each of the questions as testing specific Newtonian conceptions. The bulk of this research involved factor analysis or module analysis to find patterns among the set of Newtonian correct responses (Huffman \& Heller, 1995; Scott, Schumayer, and Grey, 2012; Scott, \& Schumayer, 2015; Brewe, et al., 2016) while ignoring the particular distractors selected by the students answering incorrectly. While this informs about what is missing, e.g. Newton's $3^{\text {rd }}$ law, it does not inform the teacher what robust beliefs (e.g. Clement, J., Brown, D. E., \& Zietsman, A. 1989) the student starts with (e.g. that a wall is inanimate and therefore cannot push back against your hand, that a moving object exerts more force than a similar object that it hits, etc.). The current work follows the smaller subset of previous research on the FCI which analyzes all 150 responses to the 30 questions and has been recently reviewed by Stewart et. al Stewart, J., Zabriskie, C., Devore, S., \& Stewart, G. (2018).

The present work is unlike the majority of these previous studies discussed above in several ways:

1. We work with a larger sample; 17,000 students from 8 colleges as described in Table I.

2. We analyze not only the correct responses to the 30 questions on the FCI, but the responses to the 120 distractors also.

3. We use item response curves for analysis of each of these responses, classifying them both qualitatively and quantitatively.

4. We use multidimensional item response theory (MIRT) to classify students on a 2-D ability space.

5. We find a Newtonian axis as well as on a new axis that is roughly orthogonal to the Newtonian axis.

Our research is heavily based on analysis of the item response curves (IRC) for each response for each question. The IRC for a particular response is the fraction of students answering that question who select that response as a function of the Newtonian ability of students. We extend previous work where IRC's from the FCI were used to find qualitative information on what the authors termed "question effectiveness" Morris, G. A., et. al. (2012). In that work the raw total score on the FCI was used as a proxy for Newtonian ability; we use 1-diimensional Item Response Theory based on grading the 30 questions dichotomously (Newtonian/wrong) to define it. More significantly we distinguish among three qualitatively different overall IRC behaviors:

- those that increase monotonically with decreasing score,

- those reaching a maximum at an intermediate score, typically located within a standard deviation of the average score, and

- those that increase monotonically with increasing score.

Not surprisingly, we find that the Newtonian correct responses are all in the latter class.

Our main research questions are:

1. What new information is obtainable from item response curves for all responses?

2. Can we find a robust second dimension of student ability using MIRT (Multidimensional Item Response Theory)?

3. Can we find a relationship between this new axis and the qualitative nature of the IRC's?

4. Can we find a strong correlation between this 
new axis and classifications based on historical and commonsense physics beliefs?

5. What initial advice can we give instructors given the pre-test results for their students?

Our major finding is that there is another dimension besides the Newtonian axis. We find that this dimension corresponds closely with the number of intermediate responses that a student gives. We find that these intermediate distractors can often be classified as Aristotelian, Medieval, or common sense with the most common being related to Medieval ideas of impetus.

In what follows, we first describe the data, which comprises pre-test responses on all FCI questions from $17 \mathrm{k}$ students from 8 colleges. We then examine 1-dimensional item response curves and identify three types of characteristic response curves. We also use 1-dimensional item response theory, both treating each question dichotomously and each response dichotomously, and examine the structure of these item response curves. The qualitative shape of these three curves are similar, and we identify the 1-dimensional axis as Newtonian skill. Finally, we use multidimensional IRT to examine students' alternate conceptions beyond Newtonian skill. Indeed, this analysis indicates an orthogonal direction to Newtonian skill, which we identify as intermediate ability.

We expect that the results of studies like this will ultimately lead to the following:

1. Improved diagnostic questions with more finely tuned distractors

2. Using higher dimension MIRT (Hambleton, Swaminathan, \& Rogers, 1991) or clustering to delineate subsets of linked misconceptions, understand student thinking on deeper level (if there is one)

3. Developing pedagogies to specifically remediate these.

\section{DATA}

The data we used came from pre and post-testing in 8 Universities and Colleges in North America. Table 1 contains a description of the universities

\begin{tabular}{|l|l|l|}
\hline Institution Type & $\begin{array}{l}\mathbf{N}_{\text {students }} \\
(\% \text { of total) }\end{array}$ & Pre-test score \\
\hline Highly Selective 1 & $213(1 \%)$ & $0.680+-0.198$ \\
\hline Highly Selective 2 & $165(1 \%)$ & $0.661+-0.191$ \\
\hline Large Public 1 & $10039(56 \%)$ & $0.265+-0.131$ \\
\hline Large Public 2 & $1560(9 \%)$ & $0.315+-0.163$ \\
\hline Large Public 3 & $4563(25 \%)$ & $0.409+-0.181$ \\
\hline
\end{tabular}

\begin{tabular}{|l|l|l|}
\hline Medium Public 1 & $444(2 \%)$ & $0.373+-0.174$ \\
\hline Medium Public 2 & $541(3 \%)$ & $0.336+-0.157$ \\
\hline Medium Private & $502(3 \%)$ & $0.427+-0.189$ \\
\hline TOTAL & 17827 & $0.322+-0.172$ \\
\hline
\end{tabular}

Table 1. Table caption

The data were preprocessed following these three criteria:

1. We only evaluate students who completed the full course. Students who dropped out were discarded from the study.

2. Students who retook the course later are considered new independent instances. This decision was made because we don't have any information on the prior courses taken by the students.

3. Any question with zero or two responses is considered incorrect.

\section{THE NEWTONIAN DIMENSION}

A student's overall score on the FCI is a measure of what we call Newtonian skill, since the correct responses are those that highlight different aspects of Newtonian thinking as embodied by Newton's three laws of motion and force, and associated ideas like instantaneous velocity and acceleration.

\section{1-Dimension Item Response Curves}

Our data were displayed as IRCs (Item Response Curves) simply by binning the probability of each response, $r=A$ $\rightarrow$ E (Fig. 1 top) according to overall score, with an error estimated using the standard deviation of a binomial distribution for each response, $r$, given the total number of students in that score bin who answered that question: $\delta_{r}=\sqrt{N_{\text {responses }} p_{r}\left(1-p_{r}\right)}$ where $p_{\boldsymbol{r}}=N_{\boldsymbol{r}} / N_{\text {responses. }}$

Our IRC's are generally quite similar in shape to those in Morris, G. A., et. al. (2012) except for having smaller errors due to our larger sample size. Those authors suggested that IRC's provide a better qualitative analysis than the item parameters determined by item response theory (IRT), principally because they give a function of ability for all 5 responses for each question rather than only a small set of parameters. More significantly, we observe many IRC's that are not fit even qualitatively by the sigmoidal (usually logistic) curves used in IRT.

\section{Three Types of Response Curves}

There are three distinct shapes that characterize most of the observed response curves that attract over $\sim 20 \%$ of the total responses to a question. See Fig 1 below: 

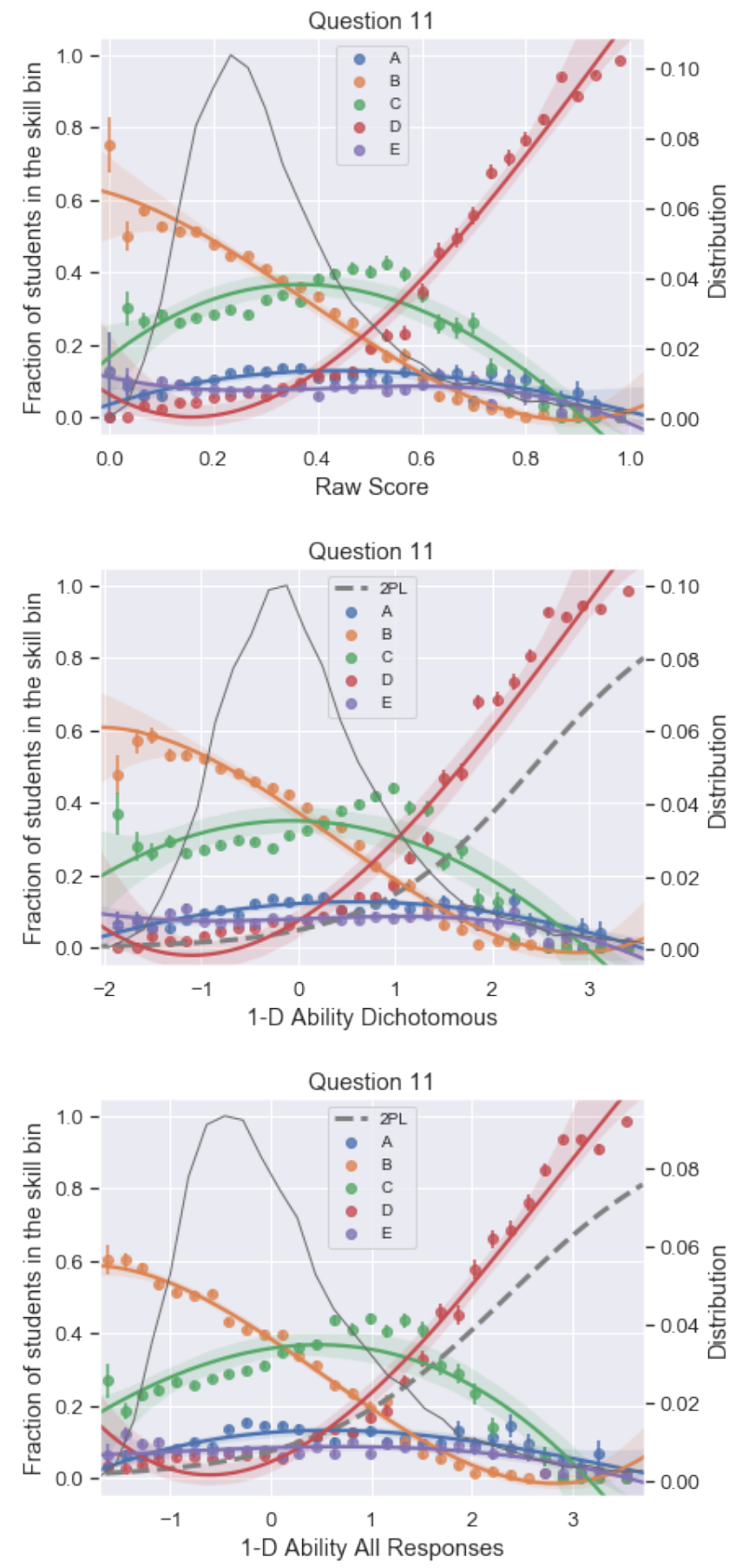

Figure 1. Response Curves for each Response on Question 11 vs. overall raw score (top), vs. 1-D IRT ability treating each question as having a dichotomous response (middle), and vs. 1-D IRT fit treating all responses as independent questions (bottom). Thin line shows distribution of students, and dashed line is IRT Logistic function fit to Newtonian Correct response. The colored lines are fits to the data using a 3rd order polynomial to guide the eye. The colored zone around each colored line is the error in the fit inferred from a bootstrap technique. dominant correct - a response that over $50 \%$ of the highest ability students select, or that a plurality selects and that increases significantly with ability at the highest ability e.g. response D (red) in Fig. 1. This behavior results in a positive discrimination parameter for that response.

intermediate maximum - a response that reaches a maximum value over $20 \%$ within the ability range -1 to +1 - e.g. response C (green) in Fig 1. Such response curves lead to small discriminations using IRT (see below).

dominant incorrect - a response that over $50 \%$ of the lowest ability students select, or that a plurality selects and that rises significantly as ability decreases at the lowest ability - e.g. response B (orange) in Fig. 1. Such a response curve has a negative discrimination.

\section{1-D IRT For Each Question Treated Dichotomously}

We then fit the response for each of the 30 questions with a one-dimensional 2-PL logistic model, which has no guessing parameter, using the "mirt" package in the " $\mathrm{R}$ " statistical software system. We refer to this model as "dichotomous" to contrast it with the models we use in the rest of the paper, which consider the specific incorrect responses separately. In the dichotomous model, the Newtonian correct response (i.e. the selection consistent with Newtonian physics) is graded 1 and all other responses as $0-$ i.e. are considered as equally counterindicative of Newtonian ability. We treated "no response" as incorrect. The response data for each of the 150 responses on the FCI are then binned according to the dichotomous IRT ability of each student and plotted in Fig. 1 (middle). This procedure yields parameters for the difficulty and discrimination (propensity for higher ability students to answer correctly) of each question, together with an ability for each student.

Figure 1 (middle) also shows the IRT fit for the Newtonian correct response. The fit on this problem would clearly be better if the difficulty were lowered, shifting the curve to the left. However, changing any of the item parameters would change the ability estimated for all students and would presumably decrease the overall likelihood of the global fit (the IRT package maximizes likelihood). Note that model is fit to all 30 questions, but we are only showing one in Fig 1.

\section{1-D IRT For Each Response Individually}

Subsequently (Fig 1 bottom), we fit all 150 responses using IRT, each considered as an independent dichotomous response item and ignoring "no response" - i.e. we do not consider the absence of a response to a question as a separate response category. A student's response data would look like $23 \mathrm{~A}=0,23 \mathrm{~B}=1,23 \mathrm{C}=0,23 \mathrm{D}=0,23 \mathrm{E}=0$, if they selected response " $\mathrm{B}$ " to question 23 . With this approach, a response of 1 no longer implies Newtonian correct, and the IRT program is simply trying to predict the 
probability of the observed responses by assigning item parameters for each response (difficulty and discrimination) and an ability for each student. We note that this procedure gives a rough fit for a dominant wrong response like 11B (with negative discrimination), but a qualitatively poor fit for a response like $11 \mathrm{C}$ which does not rise monotonically at either high or low ability.

\section{Critique and Newtonian Axis Definition}

From a psychometric perspective,

1. IRT assumes independence of the items, and it is clear that a cluster of questions that refer to the same figure or situation (e.g. 5\&6, 9-11, etc.) will generally have interdependent responses as noted by Stewart, J., Zabriskie, C., Devore, S., \& Stewart, G. (2018).

2. Item independence is strongly violated when applying IRT to all distractors for a single question as a student cannot select more than one response per question. Using Bock's graded response model would be an improvement as it handles this constraint naturally, and has been suggested. We did not pursue this because it's multi-dimensional generalization (next section) does not allow different responses to the same question to load differently on the multiple dimensions.

3. It is clear that the 1-D IRT fits to the correct answer do not fit the data very well, as seen in Fig 1 and in many of the other 30 questions.

4. On Fig 1, there is a sharp maximum and then rapid drop in response $\mathrm{C}$ accompanied by a rapid rise in response $\mathrm{D}$ (correct) around 0.6 in the raw score (18/30 answers correct). Similar structure, also well above the level of significance, is observed on both questions 4 and 13 around raw score $\sim 0.6$.

Nevertheless, our procedures produce clearly specified fits, and in addition pass the test that they give very highly similar results (note the high degree of similarity of the various curves in Fig 1).

In the following we use the 1-D dichotomous response model to define the Newtonian axis. Thus, in seeking a continuous variable that measures the 'Newtonian correctness' of a response, we would use the average 1-D dichotomous ability of the students who chose that response.

We have combined data from the 8 different institutions. This is justified by Figure 3 below which shows that although the three cohorts of each institution have widely varying initial Newtonian ability (as well as religious beliefs, part of North America, social class, etc.) there is little systematic variation on dimension two (defined in next section) among the different institutions once overall Newtonian ability is considered.

\section{ANALYSIS OF 1-D RESPONSE CURVES}

Behavior

Several conclusions are apparent from examining IRC's from all questions on the FCI.

1. In every case the Newtonian correct response is 'dominant correct' whether plotted versus the total score or the 1-D IRT dichotomous ability. In fact, on only question (15) does this response fail to exceed $80 \%$ at the highest ability.

2. About $1 / 2$ of the questions have at least one 'intermediate maximum' response. As we shall show, these often represent misconceptions that are in accord with Medieval physics beliefs.

3. There is a dominant wrong (e.g. response $\mathrm{B}$ in Fig 1) on all questions except 8, 18, 20, 22, 26, and 28 .

4. The qualitative shapes of the IRCs are similar regardless of whether they are produced using raw score, dichotomous IRT, or all-response IRT.

5. The IRT logistic curve does not fit the Newtonian correct response 11D very well; we attribute this to the fact that the IRT is a global fit to all questions and changing the item parameters for $11 \mathrm{D}$ would likely make changes in skill parameters that would make the overall likelihood lower.

\section{Conclusions From 1-D Response Curves}

Several conclusions follow from these data:

1. The dominant wrong response curves generally continue to rise continuously even below the raw score (6) that would result from random guessing. This refines the widespread conclusion that very naïve students do not guess; rather it is clear that for many questions one distractor appeals to a majority of such students

2. Not all wrong answers are equally wrong; the intermediate responses are mostly maximum in the ability range of -1 to 1 which contains about $2 / 3$ of the students.

\section{THE SECOND DIMENSION}

The main thrust of our research is to determine or classify a student's alternate conceptions of force and motion from their responses to the FCI. This must be based on the distractors they select. Taking the first axis of student ability to by the Newtonian ability described above, we find a second dimension. The essential questions are, "Can we find systematic differences in the pattern of distractors selected by students beyond the knowledge given by knowing their Newtonian ability?", and "What distinct 
alternate conceptions are associated with this second dimension?" We find that such a second dimension can be obtained by applying two dimensional MIRT analysis either to the set of all responses or just to the set of distractors (Newtonian incorrect responses). We posit that students have specific non-Newtonian belief systems or schema, that can be evidenced by responding similarly to a particular set of distractors.

We found a second dimension by applying two dimensional MIRT (Multidimensional item response theory) to all responses in our data set on the FCI. Since our ultimate objective is to determine the mental state of the students that future instruction should address, we have concentrated solely on the pretest results.

\section{2-D MIRT}

The MIRT models were fit using the "mirt" package in the "R" statistical software system. All 150 dichotomous responses were included, the one correct response and the four incorrect responses for each of the 30 items. MIRT extends unidimensional IRT by fitting multiple (k) latent ability traits, $\theta_{k i}$, for each student. For this work, two ability traits are fit for each student. MIRT predicts the probability, $\pi_{i j}$, of student $\mathrm{i}$ selecting response $\mathrm{j}$ of each of the 150 responses. The probability is modeled by the logistic function:

$$
\pi_{i j}=1 /\left(1+\exp \left(-\left[\alpha_{1 j} \cdot \theta_{1 i}+\alpha_{2 j} \cdot \theta_{2 i}+d_{j}\right]\right)\right.
$$

where $\theta_{1 i}$ and $\theta_{2 i}$ are the two dimensions of latent ability to select the response $\mathrm{j}$ for student $\mathrm{i}, \alpha_{1 j}$ and $\alpha_{2 j}$ are the discriminations of response $\mathrm{j}$ on each of the latent traits, and $d_{j}$ is the difficulty of response $\mathrm{j}$.

Like factor analysis and model-based collaborative filtering, MIRT fundamentally identifies categories that underlie clusters of responses that are typically responded to similarly by a cluster of students (e.g. a majority answer yes or no similarly on each separate response). It assigns these students a positive ability in this category and these items a positive discrimination in this category if the typical response was 1 and negative discrimination if 0 . Students answering oppositely to the above set of students on those items are given a negative ability in this category. Thus, a two-dimensional MIRT program varies the two discriminations, $\left(\alpha_{1}\right.$ and $\left.\alpha_{2}\right)$ and the difficulty $d$, of each response and the corresponding two abilities of each student, $\theta_{1}$ and $\theta_{2}$, in order that the above equation will predict the entire set of observed responses with maximum likelihood. MIRT has been shown to be roughly equivalent to exploratory factor analysis for the FCI graded dichotomously (Scott, 2015) with the benefit that in addition to estimating the factor loadings, the ability parameters for each student can also be extracted (Stewart, 2019).

\section{Newtonian Ability Axis}

The $\left(\alpha_{1} * \theta_{1}+\alpha_{2} * \theta_{2}\right)$ term in the above equation is a scalar product and hence the vectors $\alpha$ and $\theta$ may be simultaneously rotated or reflected in the 2-D space without changing the predicted probabilities. It is noteworthy that the 2-D distribution is very nearly circular (and not elliptical): there is about equal variance in the vertical $\left(\theta_{2}\right)$ direction $(\sim 1.2)$ as in the horizontal $\left(\theta_{1}\right)$ direction $(\sim 1.1)$. Thus small differences in the sample can rotate these axes. Given that the FCI is designed to measure Newtonian ability, we anticipated an elliptical distribution with the longer axis corresponding with the previously identified 'Newtonian Ability' axis from 1-D IRT. Surprisingly, neither the $\theta_{1}$ nor the $\theta_{2}$ axis corresponds to the 1-D Newtonian axis, as demonstrated by Fig 2 top, where the high (low) Newtonian ability students lie at the upper right (lower left).
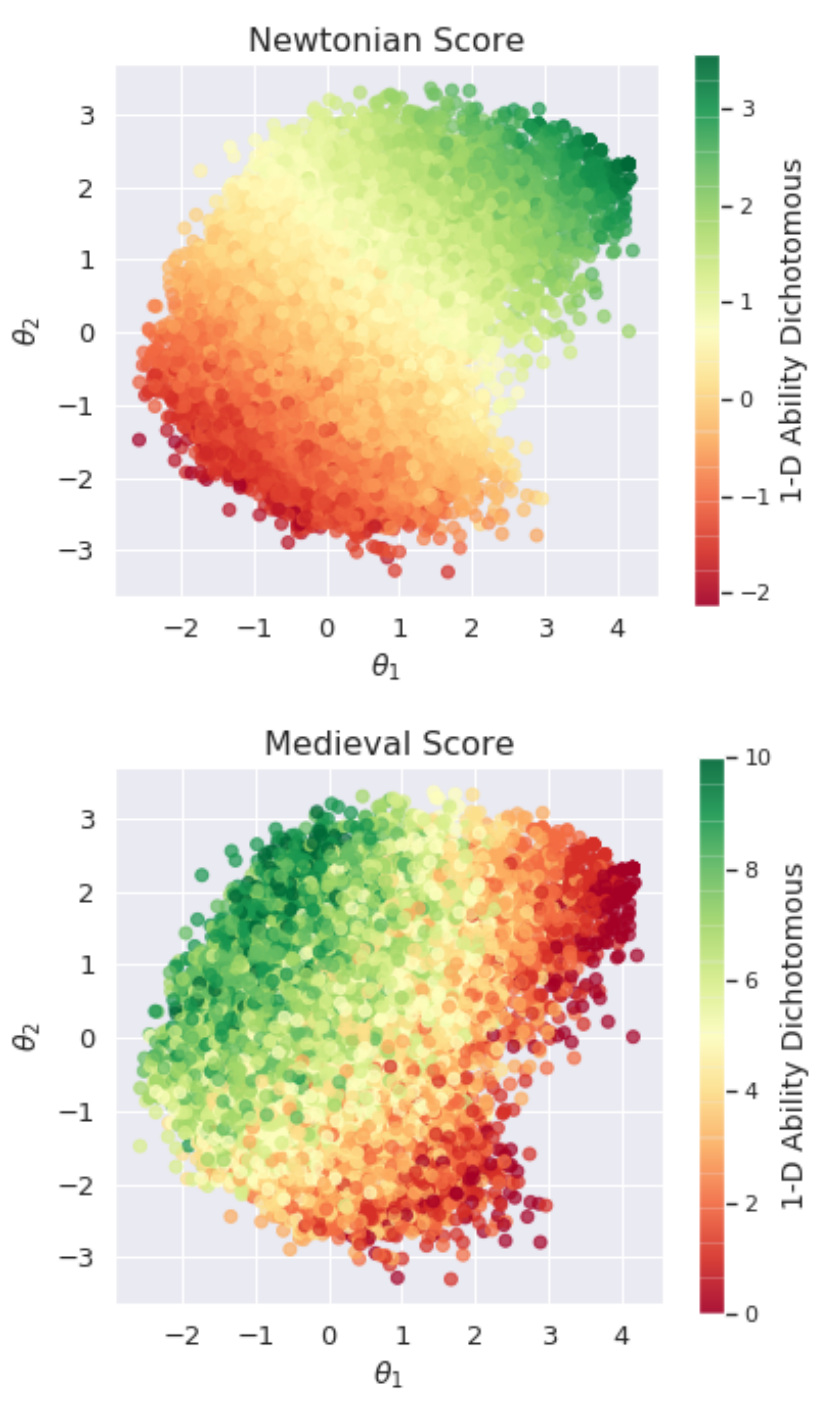
Figure 2. Each point is located on the axes $\theta_{1}, \theta_{2}$. On the top, the colors red to green represents increasing Newtonian ability; below the colors represent intermediate score which is discussed below.

The actual Newtonian axis is determined by observing where students with high Newtonian ability lie in the plane. By coloring students according to their Newtonian ability, the color gradient (top plot of Figure 2) shows that the Newtonian axis appears to lie in the direction $\left(\theta_{1}+\theta_{2}\right)$. Indeed, the variable $\left(\theta_{1}+\theta_{2}\right)$ correlates very well $(\mathrm{r}=0.98)$ with 1-D Newtonian ability (averaged over all students).

The Second Dimension is Intermediate Score

The characteristics of the other axis must be deduced by looking at the responses that are selected by students with large or small values of this dimension $\left(\theta_{2}-\theta_{1}\right)$ in conjunction with items with high discrimination along this dimension. We had previously identified 12 responses as 'intermediate maxima' (See Table 2). We then defined an intermediate score of each student as the number of intermediate responses that student selects. This is plotted in Fig. 2 bottom with green representing high intermediate score: clearly this score correlates extremely well with the $\theta_{2}-\theta_{1}$ axis.

We would expect that students with many dominant wrong responses and few intermediate responses (or a high ratio of dominant wrong to intermediate) would lie toward negative $\theta_{2}-\theta_{1}$. This explains the intriguing concave shape of Fig 2 along the negative $\theta_{2}-\theta_{1}$ boundary. Students of high Newtonian ability don't select many wrong responses; they can select dominant wrong responses for these but not enough to obtain strongly negative $\theta_{2}-\theta_{1}$. Most importantly, students with intermediate Newtonian ability are most likely to select a number of intermediate responses, which pushes them toward positive $\theta_{2}-\theta_{1}$, accounting for the 'dent' in the distribution. Finally, students with very low Newtonian ability select many more distractors, but they are less likely than those with intermediate Newtonian ability to select intermediate responses. Thus they select many more dominant wrong responses and can have the most negative $\theta_{2}-\theta_{1}$

The large vertical extent of the distribution in the $\theta_{2}-\theta_{1}$ direction indicates that this new dimension measures how students differ even though they obtained the same raw score based on Newtonian correct responses. The point is that the new dimension of the wrong answers selected by such a student can be weighted towards intermediate maximum or dominant wrong responses, and the great width of the distribution along $\theta_{2}-\theta_{1}$ shows that there is clearly a wide variation in the tendency.

\section{DISCUSSION}

In summary our 2-D analysis of all 150 responses to the FCI contains two principal orthogonal coordinates:

1. a Newtonian axis defined as the skill determined by the 1-D analysis of the FCI based on dichotomously grading each question.

2. a second roughly orthogonal axis corresponding to the number of responses with item response curves that are intermediate in nature vs the number of dominant wrong responses.

Thus a student with a particular Newtonian ability can be further classified on the basis of how many responses they give whose IRC's are intermediate vs how many are dominant wrong.

\section{Variation with University}

We next investigate how the pre-tests of students at the various schools studied depend on the university. Of course, highly selective universities will have higher Newtonian ability. The real question is whether, at a given Newtonian ability, will they have the same $\theta_{2}-\theta_{1}$.

To study this, we broke each university's students into three equal cohorts and computed the average of $\theta_{2}$ and $\theta_{1}$ for each cohort. The results are shown in Fig 3.

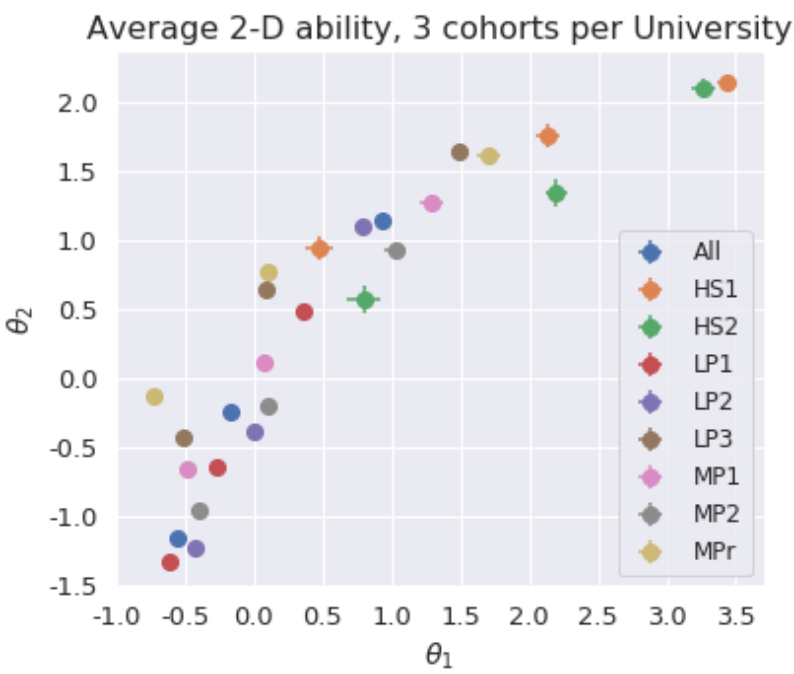

Figure 3. Institutional Independence. Students from each college (colored) in Table I are divided into 3 equal cohorts whose mean and its deviation are plotted.

The dominant feature of Fig 3 is that the points seem to lie close to a 'universal curve' in $\theta_{2} \times \theta_{1}$ space. The deviation from a 'best-fit' is statistically significant, but we see nothing systematic about these differences except possibly that the medium sized private university lies above the universal curve for which we have no explanation.

Student beliefs associated with intermediate responses While the above procedures and analysis find individual responses that are selected be students with high $\theta_{2}-\theta_{1}$, 
we would like to summarize by indicating the underlying student misconceptions. This helps illuminate those student beliefs and could also help teachers address them with specific instruction. Several sets of beliefs are helpful in this regard: the first physics as set forth by Aristotle, the developments in analyzing and explaining motion in the early 14th century in Paris and London, and especially more recent studies of students thinking and common sense - but non-Newtonian - beliefs about Mechanics.

Modern studies of alternative conceptions about mechanics include (Clement, 1982 and 1989). These and other studies ultimately led to the landmark paper Common sense concepts about motion by Halloun and Hestenes (1985b), a summary of historic ideas and current research on alternate conceptions, culminating with taxonomy of "common sense concepts" of Newtonian mechanics developed by Halloun and Hestenes (1985a \& 1985b). Items that probed such non-Newtonian concepts, commonly referred to as "misconceptions" or "alternate conceptions", were developed as distractors in the FCI. These include confusion between velocity and acceleration, various impetus ideas, the idea that forces require an active agent, and misunderstandings of action/reaction force pairs (i.e. Newton's 3rd Law) and the concept of gravity. For the complete list of student misconceptions of the FCI and which items probe each misconception, see Hestenes, Wells, \& Swackhamer (Hentenes, 1992). During the development of the FCI, the original version of the FCI was modified in 1995 (Halloun, Hake, and Mosca, 1995--HHM95); the 1995 version was used throughout this study.

\begin{tabular}{|l|l|l|}
\hline Responses & Description & Class \\
\hline $4 \mathrm{~A}, 15 \mathrm{C}, 28 \mathrm{D}$ & $\begin{array}{l}\text { moving or larger object } \\
\text { exerts more force }\end{array}$ & $\mathrm{p}$-Prim \\
\hline $\begin{array}{l}11 \mathrm{C}, \quad 13 \mathrm{C}, \\
30 \mathrm{E}\end{array}$ & $\begin{array}{l}\text { force like impetus in the } \\
\text { direction of motion }\end{array}$ & Impetus \\
\hline $17 \mathrm{~A}, 25 \mathrm{D}$ & $\begin{array}{l}\text { force exceeding the } \\
\text { resistance is necessary } \\
\text { for motion }\end{array}$ & Aristotelian \\
\hline $5 \mathrm{D}, 18 \mathrm{D}$ & $\begin{array}{l}\text { force like circular } \\
\text { impetus }\end{array}$ & Impetus \\
\hline $5 \mathrm{E}, 18 \mathrm{E}$ & $\begin{array}{l}\text { circular impetus force } \\
\text { and centrifugal force }\end{array}$ & Impetus \\
\hline
\end{tabular}

Table II The answers identified as intermediate that also grouped together using their tetrachoric correlation with each other $(4 \mathrm{~A}$ is wrong but was included due to strong correlations and because it was the most popular response. They are categorized by a description of the response, and then by the historical context of this belief. Clearly the majority are Medieval Impetus forces (Iii et al., 2018)

\section{ConcLusion}

We have shown that large data sets can be processed to give Item Response Curves that reveal qualitative features of all responses, both correct and incorrect (distractors). Where the distractors are developed based on research we've shown that analyzing the distractors provides additional insight into the students' belief systems. We applied these techniques to the FCI, in which the distractors were based on historical physics beliefs as well as research into student responses. Importantly, we showed that not all distractors were 'dominant wrong' - that is increasingly likely to be selected by students of decreasing Newtonian ability. Instead a number of frequently selected distractors are more likely to be selected by students of intermediate ability then either those of higher or lower ability. These results suggest that incorrect responses given by students result from strongly held alternate conceptions, and furthermore that students with similar levels of Newtonian understanding may hold systematically distinct sets of alternate conceptions.

The ability to identify patterns of misconceptions in students can improve learning in a number of ways. First, teachers can give their students research validated diagnostic assessments like the FCI at the beginning of a course and then tailor their instruction to the specific misconceptions exhibited by their students. Research has shown Sokoloff, D. R., \& Thornton, R. K. (1997) that instruction which specifically and explicitly confronts specific misconceptions is more effective than instruction based solely on presenting correct conceptions. The analysis we have done here will allow us to create grading schemes for the FCI and related instruments which do not simply reflect the level of Newtonian understanding displayed by the students but also measure the extent to which students hold specific alternate conceptions, such as medieval ideas of impetus or animistic thinking.

Additionally, application of these techniques to pre- and post-instruction administrations of the same concept inventory can provide valuable insight into the effectiveness of an instructor or an intervention at addressing specific alternate conceptions. Simply knowing what fraction of students successfully learned a target concept from an experimental intervention does not give enough information to effectively improve or augment that intervention. Being able to identify which alternate conceptions are particularly susceptible or resistant to an intervention will allow for more efficient targeting and refinement of instructional techniques.

Finally, these techniques will enable researchers to directly study learning progressions (Alonzo \& Aufschnaiter, 
2018), not only as students advance from misconceptions to correct conceptions, but also as they may progress through a hierarchy of different misconceptions as they advance toward full understanding. Learning progressions for the FCI have been proposed (Neumanna, Fulmerb, \& Liangc, 2013) and the application of the techniques described in this paper to data from learning at scale may allow for experimental verification or refinement of these models as well as an evaluation of whether instruction can and should skip over some intermediate levels.

\section{ACKNOWLEDGMENTS}

Financial support for this research was provided by MIT's office of Digital Learning, the MIT Physics Department, and by the NSF. Neither MIT nor NSF has endorsed the findings of this research.

We are grateful to N. Lasry and M. Dugale for deidentified FCI pre and post data from several universities.

We thank Y. Bergner and J. Shen for helpful discussions.

\section{REFERENCES}

Alonzo, A. C., \& Aufschnaiter, C. Von. (2018). Moving Beyond Misconceptions:, 470. http://doi.org/10.1119/1.5055332

Brewe, E., Bruun, J., \& Bearden, I. G. (2016). Using module analysis for multiple choice responses: A new method applied to Force Concept Inventory data. Physical Review Physics Education Research, 12(2), 020131.

Disessa, A. A., \& Sherin, B. L. (1998). What changes in conceptual change?. International journal of science education, 20(10), 1155-1191.

Clement, J. (1982). Students' preconceptions in introductory mechanics. American Journal of physics, 50(1), 66-71.

Clement, J., Brown, D. E., \& Zietsman, A. (1989). Not all preconceptions are misconceptions: finding 'anchoring conceptions' for grounding instruction on students' intuitions. International journal of science education, 11(5), 554-565.

Gupta, A., Hammer, D., \& Redish, E. F. (2010). The case for dynamic models of learners' ontologies in physics. The Journal of the Learning Sciences, 19(3), 285-321

Hake, R. R. (1998). Interactive-engagement versus traditional methods: A six-thousand-student survey of mechanics test data for introductory physics courses. American Journal of Physics, 66(Md), 126. Retrieved from http://scitation.aip.org/content/aapt/journal/ajp/66/1/ $10.1119 / 1.18809$
Halloun, I. A., \& Hestenes, D. (1985a). The initial knowledge state of college physics students. American journal of Physics, 53(11), 1043-1055.

Halloun, I. A., \& Hestenes, D. (1985b). Common sense concepts about motion. American journal of physics, 53(11), 1056-1065.

Halloun, I. A., \& Hestenes, D. (1987). Modeling instruction in mechanics, 55(August 1984), 455462.

Halloun, I., \& Hestenes, D. (n.d.). The search for conceptual coherence in FCI data. Retrieved from http://modeling.asu.edu/R\&E/CoherFCI.pdf

Hambleton, R. K., Swaminathan, H., \& Rogers, H. J. (1991). Fundamentals of item response theory. Newbury Partk, CA: Sage Publications.

Hammer, D. (1996). Misconceptions or p-prims: How may alternative perspectives of cognitive structure influence instructional perceptions and intentions. Journal of the Learning Sciences, 5(2), 97-127.

Heller, P., \& Huffman, D. (1995). Interpreting the force concept inventory: A reply to Hestenes and Halloun. The Physics Teacher, 33(8), 503. http://doi.org/10.1119/1.2344279

Hestenes, D., Wells, M., \& Swackhamer, G. (1992). Force concept inventory. The physics teacher, 30(3), 141158.

Huffman, D., \& Heller, P. (1995). What does the force concept inventory actually measure?. The Physics Teacher, 33(3), 138-143.

Iii, B., Thijssen, J. M. M. H., Science, S. E., Studies, I., Science, E., \& Murdoch, J. E. (2018). The Debate over the Nature of Motion: John Buridan, Nicole Oresme and Albert of Saxony . With an Edition of John Buridan' s " Quaestiones super libros Physicorum , Stable URL: https://www.jstor.org/stable/20617783

Mazur, E. (1999). Peer Instruction: A User's Manual. American Journal of Physics, 67(4), 359. Retrieved from

http://scitation.aip.org/content/aapt/journal/ajp/67/4/ $10.1119 / 1.19265$

Morris, G. A., Harshman, N., Branum-martin, L., Mazur, E., Mzoughi, T., Baker, S. D., ... Baker, S. D. (2012). An item response curves analysis of the Force Concept Inventory An item response curves analysis of the Force Concept Inventory, 825(March 1995). http://doi.org/10.1119/1.4731618

Neumanna, I., Fulmerb, G. W., \& Liangc, L. L. (2013). Analyzing the FCI based on a Force and Motion Learning Progression Analyzing the FCI based on a Force and Motion Learning Progression, (July). 
Sokoloff, D. R., \& Thornton, R. K. (1997). Using interactive lecture demonstrations to create an active learning environment. In AIP Conference Proceedings (Vol. 399, pp. 1061-1074). AIP. http://doi.org/10.1063/1.53109

Scott, T. F., \& Schumayer, D. (2015). Students' proficiency scores within multitrait item response theory. Physical Review Special Topics-Physics Education Research, 11(2), 020134.

Scott, T. F., Schumayer, D., \& Gray, A. R. (2012). Exploratory factor analysis of a Force Concept Inventory data set. Physical Review Special TopicsPhysics Education Research, 8(2), 020105.

Stewart, J., Zabriskie, C., Devore, S., \& Stewart, G. (2018). Multi-Dimensional Item Response Theory and the Force Concept Inventory, 26506, 1-19.

Swackhamer, G., Hestenes, D., \& Wells, M. (1992). Force concept inventory. The Physics Teacher. http://doi.org/10.1119/1.2343497 